\title{
TELEATENDIMENTO NO ENFRENTAMENTO À COVID-19
}

\section{CALL CENTER IN THE CLASH AGAINST COVID-19}

\section{Resumo}

A pandemia causada pela Covid-19 exigiu novas formas de atendimento à saúde. O "Programa UEPG de Apoio Institucional para Ações Extensionistas de Prevenção, Cuidados e Combate à Pandemia do Novo Coronavírus" foi criado na perspectiva de atender, orientar e esclarecer a população. Este Programa tem apoio financeiro da Superintendência Geral de Ciência, Tecnologia e Ensino Superior, Secretaria de Saúde e Fundação Araucária de Apoio ao Desenvolvimento Científico e Tecnológico do Estado do Paraná, sendo desenvolvido em parceria com sete universidades estaduais e a Universidade Federal do Paraná. O Programa atua em três direções: atendimento em central de informações, call center; atuação junto às regionais de saúde; e atenção às divisas rodoviárias do Estado do Paraná. Objetiva-se aqui relatar a experiência vivenciada pela equipe do call center, importante ferramenta social de teleatendimento, com escassez de produções científicas. O teleatendimento constitui-se como ferramenta de acesso rápido e simples pelas diversas formas de contato, seja por smartfone, computador e tablet e, com inúmeras formas de interação nas redes sociais, chat, chamada de voz e vídeo, atinge diferentes classes sociais e faixa etária.

Palavras-chave: Teleatendimento; Covid-19; Ferramenta social.

Data recebimento: $17 / 07 / 2020$

Data de aceite: $02 / 12 / 2020$

\begin{abstract}
The pandemic caused by Covid-19 has demanded new forms of health care. The Ponta Grossa State University (UEPG, Portuguese acronym) Institutional Support Program to Outreach Actions of Prevention, Care and Response to the Pandemic of the New Coronavirus" was created in order to answer, clarify and guide the population. The program is financially supported by the General Superintendence of Science, Technology and Higher Education, the Secretariat of Health and by the Araucária Foundation of Scientific and Technological Development Support of the State of Paraná. The program is developed in partnership with seven state universities and the Federal University of Paraná. The program acts on three aspects: information centers, call centers working alongside local health units and attention to the Paraná borders. This article reports the experiences of call centers' team, an important social tool in call centers, which are under studied. Call centers are fast and easy access tools as they use various forms of contact such as smartphones, computers, tablets as well as numerous forms of interaction in the social networks, for example, chats, voice and video calls to reach different social classes and age groups.
\end{abstract}

Keywords: Call centers; Covid-19; Social tool.
* Professor da Universidade Estadual de Ponta Grossa (UEPG), Ponta Grossa - PR, Brasil. E-mail: coradassi@gmail.com ** Professora da Universidade Estadual de Ponta Grossa (UEPG), Ponta Grossa - PR, Brasil. E-mail: fpmansani@gmail.com

*** Aluna de Graduação da Universidade Estadual de Ponta Grossa (UEPG), Ponta Grossa - PR, Brasil. E-mail: gabenassi7@gmail.com **** Professora da Universidade Estadual de Ponta Grossa (UEPG), Ponta Grossa - PR, Brasil. E-mail: lisleipreuss@gmail.com ***** Professora da Universidade Estadual de Ponta Grossa (UEPG), Ponta Grossa - PR, Brasil. E-mail: pollyannakassia@hotmail.com ****** Professor da Universidade Estadual de Ponta Grossa (UEPG), Ponta Grossa - PR, Brasil. E-mail: zanettigomes65@gmail.com 


\section{Introdução}

A tecnologia é a chave para o avanço de uma sociedade. Especificamente na área da saúde, as inovações garantem melhoria da qualidade de vida, ajudam a reduzir gastos, aceleram os exames, aperfeiçoam os métodos diagnósticos, aumentam as opções terapêuticas, impactando positivamente na morbimortalidade da população (BASHSHUR, 2011). São consideradas importantes alicerces da saúde moderna, visto que, em 1960, a expectativa de vida do brasileiro era de 48 anos e, atualmente, o indivíduo que nasce no ano de 2020, 60 anos depois, viverá em média 76,7 anos, segundo dados do Instituto Brasileiro de Geografia e Estatística (IBGE, 2019).

Tal longevidade só se tornou possível devido à evolução socioeconômica, bem como a um melhor acesso da população à rede de saúde mais modernizada e tecnológica. Nesse contexto, o desafio atual é não somente garantir que os indivíduos vivam mais, mas que vivam melhor. Os avanços tecnológicos no setor da saúde vêm com o intuito de garantir não apenas a ausência de doença nas pessoas, mas, sim, auxiliar os diversos profissionais na promoção e prevenção da saúde, monitorização e vigilância, apoio diagnóstico, tratamento, esclarecimento de dúvidas e educação em saúde (BASHSHUR, 2011).

Assim, no início do ano de 2020, durante a pandemia causada pelo novo coronavírus, novas tecnologias foram incorporadas nos serviços de saúde, após se observar exponencial taxa de transmissão do vírus Sars-CoV-2, original da China, adicionado à crescente necessidade de suporte respiratório pelos acometidos pela doença, bem como notável quantidade de casos graves de pneumonia e, por fim, de óbitos (BASHSHUR, 2011; BRASIL, 2020). Foram necessárias diversas mudanças no atendimento em saúde não apenas no Brasil, mas em todos os países do mundo, dado que se teve mais de 500 mil mortes em menos de seis meses de infecção. Em situações como essa, se faz necessário planejamento e inovação para que a rede assistencial de saúde não entre em colapso (CAETANO, 2020; WHO, 2020).

Neste cenário, vários instrumentos tecnológicos foram estreados ou ampliados, durante a pandemia causada pela Covid-19, entre eles, destaca-se as centrais de atendimento, também chamados de call centers. Tal sucesso se pauta nos protocolos e decretos lançados pelo governo nacional, cujo intuito é combater ao coronavírus através do distanciamento social, isolamento dos grupos de risco e indivíduos que não operam em serviços essenciais e que possam trabalhar em casa, além da quarentena para pacientes sintomáticos e/ou contatos (BRASIL, 2020; BASHSHUR, 2011).

O termo call center, de origem na língua inglesa, faz referência a uma central de chamada, literalmente um espaço que faz e recebe ligações telefônicas como forma de resolução de problemas. Atualmente, com a vinda da internet e dos mecanismos digitais, o acesso a esses call centers é muito rápido e simples, facilitado inclusive pelas diversas formas de contato, seja por telefone, celular, computador por meio de aplicativo, redes sociais ou companhia telefônica, com inúmeras formas de interação, como chamada, chat, mídia, de modo que pessoas de diferentes classes sociais e faixas etárias possam utilizar a ferramenta. 
Desde as primeiras centrais de atendimento até as últimas criadas durante a pandemia muita coisa mudou, mas o conceito permaneceu o mesmo (BASHSHUR, 2011).

Nesse sentido, devido às restrições sociais implantadas e o risco de se expor a ambientes contaminados, a população precisava de uma linha direta com o serviço de saúde para poder sanar suas dúvidas e solicitar orientações através de serviços remotos. Entretanto, a evolução foi maior ainda, os call centers possibilitaram, também, o atendimento médico e psicológico dos pacientes e até mesmo o fornecimento de guia para a realização de exames e prescrição de receitas médicas por ofício online. Outras vantagens desse dispositivo tecnológico é que diminui o tempo de internação, reduz custos, agiliza o tempo de atendimento, minimiza a necessidade de retorno ao hospital, estreita a curva de transmissão, além de promover maior comodidade ao paciente e ampliar a capacidade de atendimento de casos graves nos estabelecimentos de saúde (BASHSHUR, 2011; CAETANO, 2020).

A telemedicina é um ramo da medicina cuja categoria está englobada dentro do conceito da telessaúde - utilização de sistemas de informação, comunicação e tecnologias dentro da área da saúde. A primeira, quando aplicada de forma responsável e eficiente, reflete no exercício das profissões vinculadas aos serviços de saúde, usa recursos de tecnologias interativas para possibilitar cuidados integrados e humanizados, aumenta o acesso dos pacientes e, com isso, melhora a logística da cadeia de saúde, como a promoção da saúde e prevenção das doenças. Dentre as possibilidades da telemedicina, reconhecida pela Organização Mundial da Saúde (OMS) desde 1990, estão disponibilizadas diversas ferramentas de prestação de serviços à distância, como teleconsulta (atendimento remoto), teleducação (aulas virtuais), telediagnóstico (análise de exames via internet), telelaudo (interpretação de imagens à distância), teleconsultoria (consultoria online), entre outros (WEN, 2018).

Visto isso, professores e estudantes da Universidade Estadual de Ponta Grossa (UEPG) ingressaram na extensão no "Programa UEPG de Apoio Institucional para Ações Extensionistas de Prevenção, Cuidados e Combate à Pandemia do Novo Coronavírus". Este Programa tem apoio financeiro da Superintendência Geral de Ciência, Tecnologia e Ensino Superior, Secretaria de Saúde e Fundação Araucária de Apoio ao Desenvolvimento Científico e Tecnológico do Estado do Paraná, sendo desenvolvido em parceria com sete universidades estaduais e a Universidade Federal do Paraná, para contribuir na orientação e no esclarecimento da população nesse momento ímpar da Covid-19. O programa tem três direções de atuação: atendimento em central de informações, call center; atuação junto às regionais de saúde; e atenção às divisas rodoviárias do Estado do Paraná.

Este artigo visa relatar as experiências vivenciadas pelos membros da equipe no atendimento em central de informações, call center, durante a pandemia do novo coronavírus na cidade de Ponta Grossa, Paraná. No município de Ponta Grossa, a central de atendimento contou com a parceria da Fundação Municipal de Saúde (FMS). A produção tem a intencionalidade também de enfatizar a importância desta ferramenta, tanto para a população quanto para os próprios participantes. Ademais, os autores intentam contribuir com as produções científicas acerca da temática abordada, posto sua escassez. 


\section{Relato de experiência}

$\mathrm{O}$ atendimento em central de informações, call center, contou com 12 bolsistas acadêmicos da UEPG dos cursos de Medicina, Odontologia e Enfermagem, no período de março a julho de 2020. A coordenação e orientação da atividade de teleatendimento aconteceu de modo multidisciplinar com docentes de: Medicina, Farmácia, Odontologia, Serviço Social e Medicina Veterinária. A UEPG se inseriu à Chamada Pública da Fundação Araucária (FA) 09/2020 e, por meio de convênio Instituição Ensino Superior e o Estado do Paraná, os professores desenvolveram o Programa e, na sequência, os estudantes se inscreveram para participar dos atendimentos no call center no dia 20 de março de 2020 e foram escolhidos, por ordem de inscrição, no dia 30 de março de 2020. E assim, iniciaram-se as ações de extensão para o enfrentamento do novo coronavírus durante a pandemia, na cidade de Ponta Grossa, no Estado do Paraná.

Inicialmente, as atividades dos bolsistas concentraram-se em torno da contextualização teórico-prática da situação atual, formação técnica a respeito do funcionamento do sistema de saúde e capacitação em relação às orientações a serem prestadas. Em seguida, realizou-se treinamento em relação às características do novo coronavírus, apresentação clínica, tempo de incubação do vírus, formas de transmissão, diagnóstico e tratamento. Além disso, compreendeu-se a rede assistencial do município, o fluxo de pacientes, os protocolos de referência/contrarreferência e os serviços de apoio. Enfim, esses conhecimentos, junto à plena compreensão das possibilidades terapêuticas e de diagnóstico disponíveis, foram de suma importância para que os indivíduos atendidos recebessem os encaminhamentos necessários e os tratamentos possíveis.

Após a capacitação, os integrantes do projeto realizaram duas semanas de treinamento na central de atendimentos da Prefeitura da cidade, acompanhando o serviço com os residentes do Programa Municipal de Residência em Saúde Coletiva que já realizavam esse tipo de atendimento, mas de menor amplitude à população. Assim, os alunos puderam praticar os conceitos adquiridos anteriormente, simular situações, lembrar fluxos e rever condutas aprendidas. Esse momento foi crucial no acompanhamento dos residentes multiprofissionais da residência em saúde coletiva, para a troca de saberes e experiências nesse período excepcional de saúde mundial.

Finalmente, iniciaram-se os atendimentos, via telefônica, de pacientes do município, na sede da Fundação de Apoio ao Desenvolvimento Institucional, Científico e Tecnológico da Universidade Estadual de Ponta Grossa (FAUEPG), cujo serviço foi disponibilizado para a população de segunda-feira a domingo, das 8 às 22 horas. Essa assistência foi possibilitada pelo software de chamada telefônica, desenvolvido por dois técnicos em informática, os quais habilitaram toda a equipe para uso e preenchimento de dados em relação à população assistida no sistema do computador.

A equipe, inicialmente formada pelos 12 bolsistas e seus coordenadores, foi ampliada à medida que surgiu uma parceria entre a universidade e a FMS, que, por meio da residência multiprofissional em saúde coletiva, produziam atividades semelhantes às ofertadas pelo 
projeto da FA e que também atendiam a Terceira Regional de Saúde do Estado do Paraná. Nesse sentido, os call centers se uniram para intensificar as ações propostas no atendimento às demandas da população ponta-grossense no contexto da pandemia, sendo que, os bolsistas e residentes de diferentes áreas profissionais, como Educação Física, Enfermagem, Farmácia, Medicina, Medicina Veterinária, Odontologia, Psicologia e Serviço Social, se revezavam nos atendimentos em escalas presenciais.

Ademais, durante o acolhimento telefônico do paciente, caso houvesse identificação de situações de sofrimento e sinais de ansiedade e/ou depressão provocadas ou desencadeadas pelo isolamento social, o paciente era encaminhado para acolhimento psicológico, via remota, que ocorria no call center de segunda a sexta-feira, das 9 às 21 horas. Se fosse percebida a necessidade de atendimento médico, era agendado pela plataforma de consultas online para que o médico de plantão entrasse em contato durante o horário comercial, das $8 \mathrm{~h} 30 \mathrm{~min}$ às 17h30min, de segunda a sexta-feira, ou, então, era orientado a procurar a Unidade Básica de Saúde (UBS) mais próxima.

No decorrer dos atendimentos, quando necessário, era orientado aos pacientes com sintomas respiratórios classificados como urgentes procurarem a Unidade de Pronto Atendimento (UPA) referência em casos suspeitos/confirmados de Covid-19; aos demais casos graves de saúde, orientavam-se os pacientes a dirigirem-se ao Pronto Socorro e, tratando-se de emergência, era ligado para o Serviço de Atendimento Móvel de Saúde (SAMU). Referente aos pacientes com menos de 12 anos, gestantes ou puérperas, esses eram encaminhados ao Hospital Universitário Materno-Infantil.

Outrossim, os bolsistas participaram também do teleatendimento, através de uma plataforma desenvolvida pelo Estado do Paraná (SESA), por meio da qual o paciente enviava mensagem pelo aplicativo de WhatsApp ou acessava diretamente o aplicativo de telemedicina PR. Assim, ele podia tanto sanar dúvidas sobre questões relacionadas à pandemia, pedir informações epidemiológicas, consultar decretos e protocolos, quanto ser atendido por profissional médico e psicólogo. Além disso, através desse sistema, havia a possibilidade de enviar imagens, vídeos, documentos, encaminhamentos de áudios e realizar vídeo-chamadas ao paciente.

No Programa, foram realizadas atividades online de educação permanente, quinzenalmente, através de relatos de experiências, compartilhamento de casos/condutas - sem identificação de profissionais ou pacientes - e orientações aos estudantes pelos professores orientadores, conforme as necessidades do call center. Como forma de registro, acompanhamento, avaliação e autoavaliação das atividades realizadas para fins educativos, mensalmente eram construídos relatórios, de uso exclusivo da equipe. Assim, foi possível analisar o grau de impacto do serviço e sua contribuição para a população nesse momento de pandemia. 


\section{Resultados e discussões}

Desde o dia 01 de abril, início das atividades do call center, até o dia 10 de julho de 2020, os participantes do projeto atenderam 1.300 ligações, além de encaminhamentos para acolhimento psicológico e telemedicina. Esses últimos possibilitaram que os profissionais de saúde atendessem seus pacientes à distância, podendo, desse modo, ser considerada a evolução natural dos cuidados em saúde, proporcionando qualidade, equidade e acessibilidade de atendimento. O público atendido pelas 1.300 ligações recebidas, pela central de informações, foi composto majoritariamente de mulheres $(58,4 \%)$, mais especificamente 760 ; os homens atendidos foram $353(27,1 \%)$, e de 187 (14,3\%) pacientes não foram registrados o sexo biológico. Os dados obtidos foram coletados junto às matrizes de atendimento do call center, as quais são compostas por programa de compilação de dados desenvolvido pela UEPG, e a Plataforma Vitória ofertada para utilização via Governo do Estado do Paraná.

Salienta-se que, obedecendo às normatizações de pesquisa em saúde do Comitê de Ética em Pesquisa, este relato integra a Pesquisa Covid-19: Perfil dos Atendimentos via Plataforma Victória / Paraná e Perfil Epidemiológico dos Pacientes Atendidos nas Diferentes Regionais de Saúde do Estado do Paraná, submetida e aprovada no Comitê de Ética em Pesquisa da Universidade Estadual de Ponta Grossa / UEPG, sob parecer número 3.303.309.

No momento do atendimento pelo call center, os usuários eram questionados em relação ao isolamento, sendo que apenas $238(18,3 \%)$ pacientes afirmaram estar praticando o isolamento. A Covid-19 trouxe impactos sociais, chamando a atenção pelo seu alcance e pela velocidade com que se disseminou. Neste cenário, a prática do isolamento e distanciamento social, da quarentena e das medidas de higienização têm se constituído em principais estratégias para diminuir a curva de contágio do coronavírus e evitando o colapso do sistema de saúde, visto se tratar de um vírus altamente transmissível, que tem ocasionado um número elevado de mortes (CAETANO, 2020; BRASIL, 2020).

Estudos recentes, divulgados pelo Ministério da Saúde, mostraram relação entre a gravidade da Covid-19 e algumas comorbidades. Dessa forma, os portadores de comorbidades são denominados como grupo de risco. Durante os atendimentos na central de informações, 166 pacientes $(12,7 \%)$ declararam pertencer ao grupo de risco. Vale ressaltar que as gestantes e puérperas não fazem parte do grupo de risco para o novo coronavírus, segundo os mesmos estudos. Porém, esse grupo é associado a maiores taxas de complicações, caso sejam infectadas pelo vírus da influenza. Nesse entender, durante os atendimentos prestados no call center, somente 7 (0,53\%) mulheres se declararam como gestantes (BRASIL, 2020).

Questionado o motivo da ligação para o call center, a maioria dos indivíduos, 660 $(50,7 \%)$, afirmou entrar em contato para saber onde procurar atendimento de saúde presencial. Outros determinantes citados foram: orientações em relação ao isolamento e distanciamento social $(15,55 \%)$, denúncia $(8,96 \%)$, depressão/solidão durante o isolamento $(6,16 \%)$, ansiedade $(6,86 \%)$, dúvidas sobre a situação epidemiológica da região $(7,14 \%)$, reclamações $(3,78 \%)$ e perguntas sobre vacina da gripe $(0,84 \%)$. 
Salienta-se que 169 pessoas que procuraram o serviço por apresentarem problemas psicológicos originados ou agravados devido à pandemia foram encaminhadas para atendimento psicológico, totalizando $13,02 \%$ das ligações. As pessoas que ligaram para prestar queixa, denunciar alguém ou alguma situação foram acolhidas e aconselhadas a retornar para o número 153 ou 156, Guarda Municipal e ouvidoria da Prefeitura Municipal, respectivamente, conforme a ocasião.

Para as pessoas que entraram em contato procurando informações no tocante à situação epidemiológica da cidade, estado ou do país, eram comunicados os dados dos últimos boletins informativos registrados. Infelizmente, com a popularização das mídias sociais e amplo acesso aos meios digitais, o mundo enfrenta um grande problema na era da informação, que são as chamadas "fake news" - notícias falsas - sendo, portanto, crucial o acesso da população a locais que forneçam notas verídicas (CAETANO, 2020).

Dos pacientes sintomáticos (660 pessoas) que ligaram para o call center, o sintoma mais comum foi mal-estar/indisposição $(21,92 \%)$, seguido de tosse $(19,5 \%)$, febre $(18,57 \%)$, dispneia (falta de ar) $(12,63 \%)$, coriza $(11,23 \%)$, congestão nasal $(8,42 \%)$ e diarreia $(7,67 \%)$. De acordo com o Ministério da Saúde (2020), os principais sintomas relacionados à Covid19 são febre, tosse seca e cansaço. Porém, cabe lembrar que o vírus afeta as pessoas de distintas maneiras, desde formas assintomáticas ou sintomas inespecíficos até formas graves da enfermidade, sendo, por isso, incapaz de se afirmar ou excluir a doença apenas pela apresentação clínica do paciente, sem a correlação epidemiológica e a confirmação laboratorial (BRASIL, 2020).

Os pacientes que ligaram no call center com sintomas graves eram orientados a procurarem o serviço de urgência e emergência do local, ao passo que os moradores da cidade eram aconselhados a se dirigirem a Unidade de Pronto Atendimento (UPA) do bairro Santa Paula ou, dependendo da gravidade do caso, ligarem para o Serviço de Atendimento Móvel de Urgência (SAMU), no número 192. Foram instruídos a procurar a Unidade Básica de Saúde (UBS) mais próxima da sua residência ou encaixados para atendimento por telemedicina os indivíduos que apresentavam caso leve ou moderado suspeito de Covid-19, bem como aqueles que necessitavam renovar receitas, atestado médico e outros quadros clínicos. Já os casos de urgência e emergência não relacionados a sintomas respiratórios eram encaminhados ao Pronto Socorro de referência e, se o público fosse composto de gestantes, puérperas ou crianças menores de 12 anos, o encaminhamento era para o Hospital Universitário Materno-Infantil.

Dos 660 doentes com sintomas, 50\% negaram ser portadores de comorbidades; $17,06 \%$ possuíam problemas cardíacos (doença não especificada, hipertensão, transplante); $8,28 \%$ relataram ter depressão/ansiedade/síndrome do pânico; $8,11 \%$ tiveram pneumonia (asma, DPOC, bronquite, enfisema); 6,08\% eram tabagistas; 6,59\% tinham diabetes, e 3,89\% apresentavam comorbidades não especificadas. Ressalta-se que a mortalidade por Covid-19 apresenta grande correlação com algumas doenças crônicas associadas e é diretamente proporcional à idade (BRASIL, 2020). 
Segundo registros do sistema telefônico do call center de Ponta Grossa, na primeira semana de abril, 51 pacientes procuraram o serviço; na segunda semana, 143; na terceira, 98; e, na quarta, 106. A grande demanda das chamadas nesse período se deu por causa da chegada da imunização contra o vírus da gripe (influenza) na cidade. Dessa forma, a população ligava à central de atendimentos em busca de informações sobre quais pessoas poderiam receber a vacina e os pontos de assistência.

No mês de maio, o número de ligações se manteve constante, sendo $75,53,55$ e, na última semana, 56, respectivamente. O número de casos no município estava controlado, de acordo com o Boletim Municipal Oficial de Ponta Grossa, disponível no site da Prefeitura Municipal da cidade. Por fim, o mês de junho iniciou com 41 ligações, seguido de um crescimento visível: 58, 89 até 198 na última semana, às custas da grande quantidade de pessoas infectadas pelo novo coronavírus, segundo dados publicados em saúde.gov.br.

Após aumento crescente, os atendimentos se mantiveram durante o início do mês de julho, sendo 147 telefonemas na primeira semana e 129 na segunda. Logo, os dados sugerem que o número de chamadas variou conforme divulgação de vacina, decretos e boletins epidemiológicos, bem como foi proporcional ao crescimento dos casos de infectados (Gráfico 1).

Gráfico 1 - Número de chamadas telefônicas por semana recebidas na central de teleatendimentos Covid UEPG/FMS. Ponta Grossa, Paraná: abril-julho, 2020

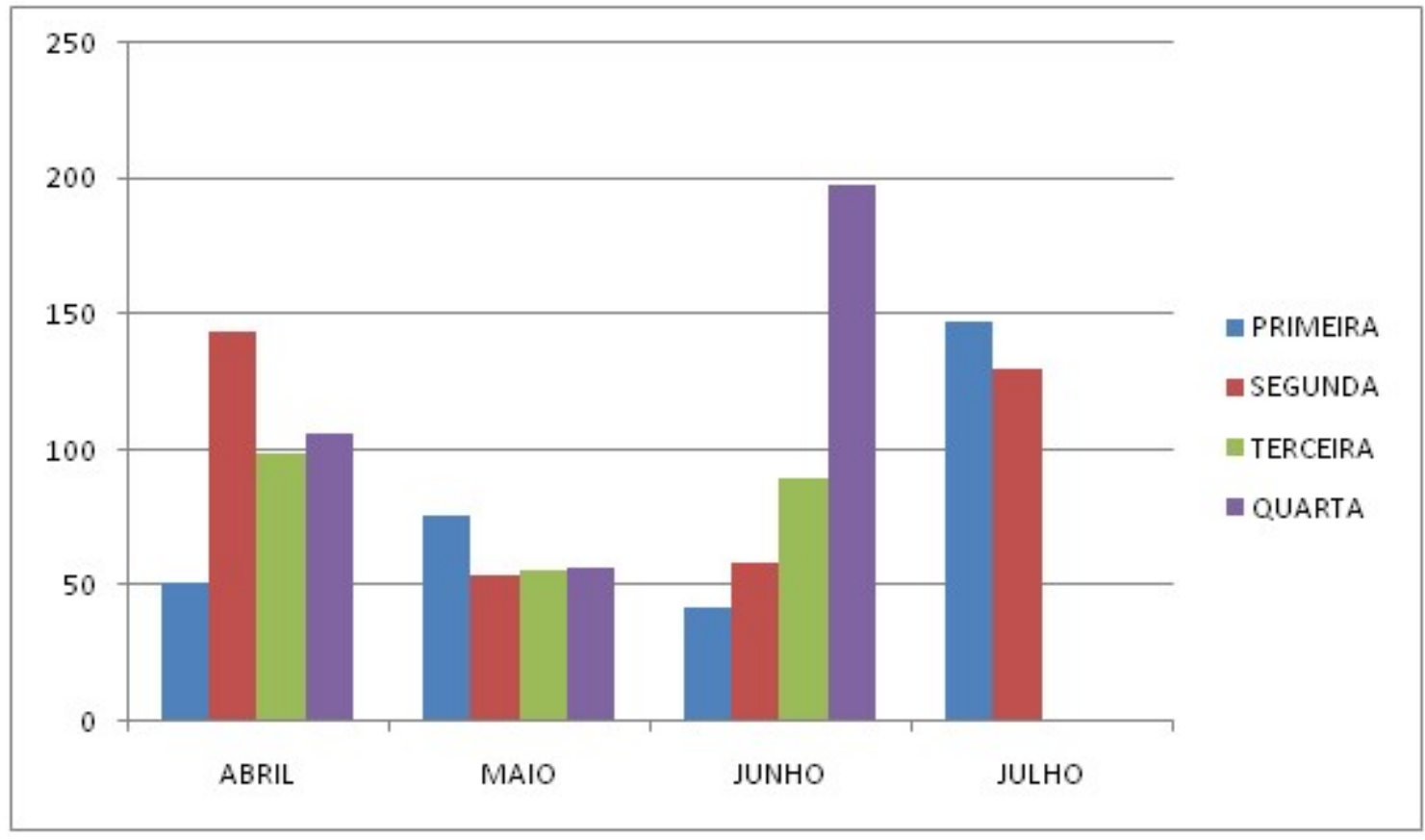

Fonte: os autores; 2020. Ponta Grossa. 
A plataforma de telessaúde foi criada exclusivamente para a assistência médica, de enfermagem e psicológica dos pacientes, de forma segura, durante a pandemia. Os atendimentos feitos através desta tecnologia sugerem a democratização do acesso à saúde, visto que todos os pacientes são atendidos e acolhidos da mesma forma pelo atendente. Além disso, a plataforma assegurou o atendimento em tempo real, redução de custos assistenciais para o governo, diminuição do risco de infecção tanto pelo profissional quanto pelo paciente, bem como possibilitou a continuidade do atendimento e retorno aberto caso o paciente precisasse.

Vale ressaltar que call center e plataforma não competem entre si, pois ambos são ferramentas que convergem para a mesma ação, sendo que um se utiliza de chamadas telefônicas como forma de contato com a população e o outro mensagens de texto, de forma que se completam.

Quinzenalmente, foram realizadas reuniões de equipe para discussão de casos e condutas, aprimoramento das tecnologias digitais, orientações sobre as plataformas e decretos, informações sobre fluxos, solicitações e leituras de exames para o novo coronavírus. Tudo isso através de vídeo chamadas, cuja tecnologia foi criada pelo homem a serviço do homem, o que contribuiu enormemente para o contato e resolução de empecilhos, via remota, nesse período crítico.

Ademais, uma das temáticas solicitadas pelos bolsistas do projeto durante os encontros de educação permanente foi a respeito da violência doméstica, assunto cujos participantes não tinham muito conhecimento e que foi uma das demandas atendidas nas ligações telefônicas durante a pandemia. Dessa forma, teve-se a participação de uma profissional, assistente social, na abordagem e sensibilização à temática, objetivando o reconhecimento, acolhimento e encaminhamento das vítimas de violência que ligam no call center em busca de ajuda.

\section{Considerações Finais}

A globalização favoreceu a rápida taxa de transmissão da Covid-19, visto se tratar de um vírus altamente transmissível. O momento atual da pandemia exige um pensamento coletivo, do isolamento e distanciamento social até a empatia e auxílio ao próximo. Só assim será possível reduzir o número de infectados e, consequentemente, de complicações e óbitos. Uma atitude solidária pressupõe a socialização de achados científicos para o tratamento e, quem sabe, vacina e cura da doença.

Nesse sentido, o mundo está interconectado pelas tecnologias desenvolvidas pelo ser humano. Portanto, nada mais coerente do que recorrer às inovações tecnológicas para a promoção e recuperação da saúde populacional. Assim, os call centers são de suma importância para esse momento de fragilidade mundial, bem como fundamentais para a socialização em saúde, via remota, como observado no projeto. 
Por fim, a central de atendimento na cidade de Ponta Grossa constituiu uma importante estratégia social, crucial para a prestação de serviço na área da saúde na pandemia da Covid19, além do acolhimento da demanda de saúde mental originada ou agravada no momento de isolamento social, da orientação de fluxos, informações epidemiológicas e consultas online. O projeto amparou positivamente seus participantes, tanto no cunho profissional, com essa experiência singular de atuação, quanto no cunho pessoal, no enfrentamento de seus próprios medos durante esse período de instabilidade, com notável influência no contexto psicossocial.

Tem-se clareza de que ainda há muito a ser desenvolvido na interface saúde e tecnologia, bem como em como efetivar a humanização nos serviços de atendimento remotos. Nesse entender, o programa de teleatendimento oportunizou a aproximação das pessoas à tecnologia em saúde de forma satisfatória e complementar à rede assistencial. Possibilitou e contribuiu, também, na formação de profissionais para o uso de tecnologias e aproximou a universidade da comunidade, mesmo em tempos de distanciamento social

\section{Referências}

BASHSHUR, R. et al. A taxonomia da telemedicina. Telemed J E Health, v.17, p.484-94, 2011.

BRASIL. Ministério da Saúde. Portaria no 188, de 03 de fevereiro de 2020. Declara Emergência em Saúde Pública de Importância Nacional (ESPIN) em decorrência da infecção humana pelo novo coronavírus (2019-nCoV). Diário Oficial da União, Brasília, DF, 4 fev. 2020.

BRASIL. Ministério da Saúde. Coronavírus Covid-19: Fast-track de teleatendimento para a atenção primária: fluxo rápido - versão 6. LILACS, coleciona SUS. 2020.

CAETANO, R. et al. Desafios e oportunidades para telessaúde em tempos da pandemia pela Covid-19: uma reflexão sobre os espaços e iniciativas no contexto brasileiro. Cad. Saúde Pública, Rio de Janeiro, v.36 2020.

INSTITUTO BRASILEIRO DE GEOGRAFIA E ESTATÍSTICA (IBGE). Tábua completa de mortalidade para o Brasil - 2018. Breve análise da evolução da mortalidade no Brasil, 2019.

WEN, C. L. Homem Virtual (Ser Humano Virtual 3D): A Integração da Computação Gráfica, Impressão 3D e Realidade Virtual para Aprendizado de Anatomia, Fisiologia e Fisiopatologia. Revista de Graduação USP, v. 1, p. 7-15, 2018.

WORLD HEALTH ORGANIZATION - WHO. Pandemia do novo coronavírus (Covid19). 2020. Disponível em: https://www.who.int/. Acesso em: 03 jul. 2020. 